\title{
Fatores associados à cura da tuberculose no Norte de Minas Gerais, 2009 a 2018
}

\author{
Factors associated with the cure for tuberculosis in Northern Minas Gerais, 2009 to 2018 \\ Factores asociados a la cura de la tuberculosis en el norte de Minas Gerais, 2009 a 2018
}

Recebido: 29/10/2021 | Revisado: 05/11/2021 | Aceito: 08/11/2021 | Publicado: 13/11/2021

\author{
Siderllany Aparecida Vieira Mendes de Brito \\ ORCID: https://orcid.org/0000-0002-2805-0750 \\ Secretaria de Estado de Saúde de Minas Gerais, Brasil \\ E-mail: siderllany.brito@ufvjm.edu.br \\ Geraldo Cunha Cury \\ ORCID: https://orcid.org/0000-0002-0411-8343 \\ Universidade Federal dos Vales do Jequitinhonha e Mucuri, Brasil \\ Universidade Federal de Minas Gerais, Brasil \\ E-mail: geraldo.cury@ufvjm.edu.br
}

\begin{abstract}
Resumo
Objetivo: Este estudo tem como objetivo analisar os fatores associados à cura da Tuberculose pulmonar no Norte de Minas Gerais/Brasil, no período de 2009 a 2018. Métodos: Estudo transversal e analítico, utilizando dados de notificações de pacientes residentes em 53 municípios registradas nos anos de 2009 a 2018 no Sistema de Informação de Agravos de Notificação compulsória (SINAN). Foram verificadas existência de associações entre a variável situação de encerramento (cura/não cura) e sexo, idade, raça, escolaridade, populações especiais, fatores de risco/comorbidades e variáveis de assistência programática, propostas em modelo teórico de eixos de vulnerabilidades. Para tanto foram realizados teste de Qui-quadrado de Pearson ou Exato de Fisher, para valores esperados menores de cinco, e por regressão logística binária calculado odds ratio e intervalos de confiança de $95 \%$ para todas as variáveis significativas $\mathrm{p}<0,20$. Resultados: foram analisados 1237 casos novos de tuberculose pulmonar que evidenciaram, neste estudo, que ser do sexo feminino, ter ensino médio completo, ser beneficiário de transferências governamentais de renda, não ser HIV positivo, não ser alcoolista, ser diabético, fazer tratamento diretamente observado aumentam as chances de cura da tuberculose pulmonar. Espera-se que o estudo contribua para estratégias que possam impactar positivamente no desfecho do tratamento da tuberculose.
\end{abstract}

Palavras-chave: Tuberculose; Cura; Norte de Minas.

\begin{abstract}
Objective: This study aims to analyze the factors associated with the cure of pulmonary Tuberculosis in the North of Minas Gerais/Brazil, from 2009 to 2018. Methods: Cross-sectional and analytical study, using data from notifications of patients residing in 53 municipalities registered in the years from 2009 to 2018 in the Information System for Compulsory Notifications (SINAN). The existence of associations between the variable status of closure (cure/noncure) and sex, age, race, education, special populations, risk factors/comorbidities and programmatic assistance variables, proposed in a theoretical model of axes of vulnerabilities, were verified. For this purpose, Pearson's Chisquare test or Fisher's exact test were performed, for expected values less than five, and by binary logistic regression, odds ratio and $95 \%$ confidence intervals were calculated for all significant variables $p<0.20$. Results: 1237 new cases of pulmonary tuberculosis were analyzed, which showed, in this sample, that being female, having completed high school, being a beneficiary of government income transfers, not being HIV positive, not being an alcoholic, being diabetic, undergoing treatment directly observed increase the chances of curing pulmonary tuberculosis. It is expected that the study will contribute to strategies that can positively impact the outcome of tuberculosis treatment.
\end{abstract}

Keywords: Tuberculosis; Cure; North of Minas.

\section{Resumen}

Objetivo: Este estudio tiene como objetivo analizar los factores asociados a la curación de la tuberculosis pulmonar en el norte de Minas Gerais / Brasil, de 2009 a 2018. Métodos: Estudio transversal y analítico, utilizando datos de notificaciones de pacientes residentes en 53 municipios registrados. en los años 2009 a 2018 en el Sistema de Información de Notificaciones Obligatorias (SINAN). Se verificó la existencia de asociaciones entre la variable estado de cierre (cura / no cura) y sexo, edad, raza, educación, poblaciones especiales, factores de riesgo / comorbilidades y variables de asistencia programática, propuestas en un modelo teórico de ejes de vulnerabilidades. Para ello se realizaron pruebas de Chi-cuadrado de Pearson o Exacto de Fisher, para valores esperados menores a cinco, y mediante regresión logística binaria se calcularon odds ratio e intervalos de confianza del $95 \%$ para todas las variables significativas $\mathrm{p}<0,20$. Resultados: Se analizaron 1237 nuevos casos de tuberculosis pulmonar, que mostraron, en este estudio, que ser mujer, haber completado la escuela secundaria, ser beneficiaria de transferencias de ingresos del 
gobierno, no ser VIH positivo, no ser alcohólico, ser diabético, estar en tratamiento directamente observado aumentan las posibilidades de curar la tuberculosis pulmonar. Se espera que el estudio contribuya a las estrategias que pueden tener un impacto positivo en el resultado del tratamiento de la tuberculosis.

Palabras clave: Tuberculosis; Cura; Norte del Minas.

\section{Introdução}

A tuberculose (TB) é uma doença infecciosa de grande importância epidemiológica que permanece como uma das mais graves ameaças à saúde pública global (OMS, 2020). Considerada emergência mundial desde 1993 pela Organização das Nações Unidas (Campos \& de Silva, 2020) resiste às várias estratégias adotadas para o controle do agravo desde então, permanecendo em condição de epidemia com uma suntuosa estimativa de 10 milhões $( \pm 8,9-11,0)$ de casos novos e 1,2 milhões $( \pm 1,1-1,3)$ de óbitos entre pessoas HIV negativos e 208 mil mortes em HIV positivos em 2019 (OMS, 2020). Está entre as dez principais causas de morte, sendo a principal causa quando considerada um único agente infeccioso (Pinto, Silveira, Rujula, Chiaravalloti, \& Ribeiro, 2017).

O Brasil está classificado entre os 22 países com maior carga de TB no mundo, e de forma propositiva teve um importante papel na elaboração da estratégia devido às experiências positivas com o Sistema Único de Saúde (SUS) e com a Rede Brasileira de Pesquisas em TB (Minas Gerais, 2019). Desde 2017 conta com o Plano Nacional pelo Fim da Tuberculose como Problema de Saúde Pública que possui metas consonantes com as globais de redução do coeficiente de incidência da doença para menos de 10 casos e redução do coeficiente de mortalidade para menos de um 1 óbito a cada 100 mil habitantes até 2035 (Brasil, 2019a). Em 2018, foram diagnosticados 72.788 casos novos de TB no Brasil, com um coeficiente de incidência de 34,8 casos/100 mil habitantes e em 2017 registrou 4.534 óbitos pela doença, o que equivale ao coeficiente de mortalidade de 2,2 óbitos/100 mil habitantes e esses dados ainda são números preocupantes (Brasil, 2019a).

Para Pinto et al. (2017) a TB é exemplo consagrado da Determinação Social do Processo Saúde-Doença. Acomete grupos de maior vulnerabilidade social e econômica, principalmente nas faixas etárias economicamente ativas gerando impactos profundos na sociedade, comunidade e famílias (OMS, 2015). Além disso, é uma doença que pode ser prevenida e curada, porém se perpetua, pois é extensamente associada aos processos de desigualdades sociais de difícil manejo (Bertolozzi, Takahashi, Hino, Litvoc, \& de Siqueira França, 2014; Brasil, 2019b).

Os fatores sociodemográficos e socioeconômicos são descritos como principais influências nos resultados dos tratamentos da TB (Andrade, Nery, Souza, \& Pereira, 2018; Santos, Sales, Prado, \& Maciel, 2018). O desfecho do tratamento da doença pode ser associado a fatores como: escolaridade, sexo, idade, renda, ocupação, apoio familiar e as comorbidades alcoolismo e HIV/AIDS (Santos et al., 2018; Valente, Ângelo, Kawa, \& Baltar, 2019).

Estudo transversal realizado no estado do Rio de Janeiro, uma das regiões de maiores cargas da doença no Brasil, no período de 2011 a 2014 analisou os fatores associados à cura no tratamento da TB a partir de 57.142 notificações no Sistema de Informação de Agravos de Notificação (SINAN) e encontrou várias associações para o desfecho da cura da TB (Santos et al., 2018). Nesse estudo, ser adulto jovem do sexo masculino, de baixa escolaridade, preto ou pardo, residir em área urbana, encontrar-se institucionalizado, ter o resultado do raio $\mathrm{X}$ de tórax suspeito, baciloscopia de primeira amostra e cultura de escarro positivas, associa-se a menores chances de cura da tuberculose. O desfecho para o tratamento da TB revelou-se associado às características clínicas e a fatores sociodemográficos, corroborando o modelo teórico de eixos de vulnerabilidades proposto no estudo (Santos et al., 2018).

San Pedro e Oliveira (2013) analisaram estudos de associação entre TB e fatores socioeconômicos oriundos de diferentes países, em revisão sistemática de literatura publicada obteve que a TB permanece intimamente relacionada às condições de vida. Tal como se vê no estudo do perfil epidemiológico da TB no estado do Piauí, nos anos de 2015 a 2020 confirmou-se que o estado é uma área com alta carga de TB, de transmissão acentuada e que a doença está associada às 
condições socioeconômicas dos indivíduos (Sousa, Mendes, Carvalho, Melo, \& de Araújo, 2021).

Um estudo randomizado realizado em 32 favelas no Peru constatou que o suporte socioeconômico no início da terapia preventiva da TB em contatos domiciliares de pacientes com TB impacta no sucesso do tratamento dos pacientes (Wingfield $e t$ al., 2017). Isso ocorre em ambos os subgrupos mais pobres e menos pobres, reforçando a ideia de que os resultados bem sucedidos são influenciados por questões socioeconômicas (Silva, Moura, \& Caldas, 2014). Frente a isso, observa-se que o sucesso do tratamento perpassa pela assistência humanizada e apta a identificar as vulnerabilidades presentes e manejá-las (Brasil 2019b).

O Ministério da Saúde, por meio do Programa Nacional de Controle da Tuberculose no Brasil (PNCT/MS) estabelece como parâmetro de cura o percentual mínimo de $85 \%$ dos casos de TB. Além disso, preconiza que a situação de encerramento por "cura" pode ocorrer por critério laboratorial ou quando não é possível por critério clínico. Dito isso, o paciente, inicialmente bacilífero, é considerado curado por critério laboratorial quando realiza no mínimo duas baciloscopias negativas, uma a qualquer tempo e outra no quinto ou sexto mês de tratamento (Santos et al., 2018). Contudo, a alta por cura também será dada ao paciente que completar o tratamento sem evidência de falência, nesse caso a alta ocorrerá com base em critérios clínicos e radiológicos, por impossibilidade de realização de exames de baciloscopia (Pinto et al., 2017).

O percentual de cura entre os casos novos de TB pulmonar com confirmação laboratorial em 2017 no Brasil foi de 71,4\% (Brasil 2019a). Dado ligeiramente inferior ao da taxa de cura encontrada em estudo realizado no Rio Grande do Sul que em média nos anos de 2006 a 2016 perfez 76\% de cura e maior que os 61 \% de cura encontrado no estado do Piauí nos anos de 2015 a 2020 (Deus, Goerch, Noal, Megier, \& Anversa, 2020; Vilela et al., 2021). Os resultados obtidos no presente estudo são semelhantes aos de Minas Gerais, que ao longo dos anos mostrou ocorrência de queda do percentual de cura, partindo de 80,2\% em 2007 para 71,2\% em 2017 (Minas Gerais, 2019).

Vale destacar que, por meio de revisão integrativa da literatura, percebe-se que existem poucos estudos que utilizaram estatísticas relacionadas aos fatores que podem se associar à cura da TB, sobretudo nos últimos cinco anos. O quantitativo de estudos encontrados evidencia lacunas deixadas pela ausência de várias possibilidades de estudos relevantes para a área da epidemiologia. Ao mesmo tempo, esses estudos são importantes para avaliar a carga e a determinação social da doença no território.

O estado de Minas Gerais é o quinto com maior número de casos no país, sendo o norte de Minas uma das regiões de maior carga de tuberculose do estado, marcado por grande vulnerabilidade social e um importante contingente populacional (Minas Gerais, 2019). Este estudo, que conta com uma série histórica de uma década, pode agregar à epidemiologia, principalmente em nível local e regional, informações úteis na implementação de políticas públicas, identificação de grupos mais suscetíveis aos desfechos desfavoráveis, sensibilização das equipes de saúde e ainda desempenhar papel relevante na formação acadêmica voltada ao cuidado centrado no paciente e comunidade e para a educação permanente dos profissionais de saúde. Além disso, existem poucos dados descritos na literatura sobre a cura de novos casos de TB notificados nessa região.

Neste contexto, este trabalho objetivou analisar os fatores associados à cura da tuberculose (TB) pulmonar dos casos novos notificados de TB de 53 municípios do Norte de Minas Gerais no período de 2009 e 2018.

\section{Metodologia}

Esse estudo é observacional, transversal, retrospectivo e analítico. Foi desenvolvido através da utilização de dados secundários do período de 2009 a 2018 extraídos do banco de dados do SINAN com uso do TABWIN. O período escolhido relaciona-se à possibilidade de construção de série histórica dos últimos 10 anos encerrados no SINAN. Considerou-se para o estudo o território jurisdicionado da Superintendência Regional de Saúde de Montes Claros/MG, no período de estudo, composto por 53 municípios na região Norte de Minas Gerais o qual apresenta população de aproximadamente 1.114.020 
habitantes e é classificado como uma região de grande extensão territorial e indicadores sociais baixos (IBGE, 2020; Minas Gerais, 2019).

Na coleta de dados, ocorrida em dezembro de 2020, inicialmente aplicado o critério de inclusão por período, de 2009 a 2018, foram recuperadas 2468 notificações, que constituem o universo trabalhado neste estudo. No entanto, foram estabelecidos critérios de exclusão para a realização do estudo sobre a cura dos casos novos de TB pulmonar conforme apresentado no fluxograma (Figura 1).

Figura 1 - Algoritmo de critérios de inclusão e exclusão.

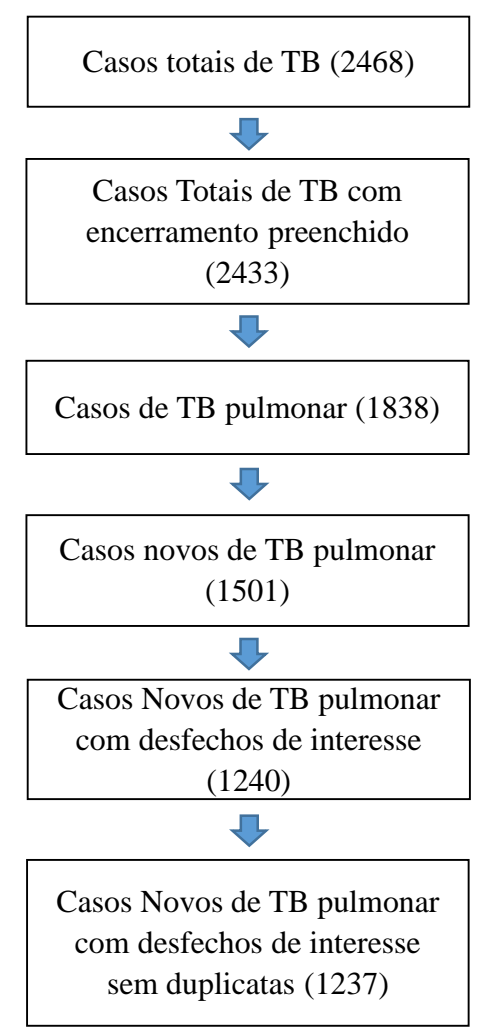

Inclusão : Período de 2009 a 2018

Exclusão : Encerramento não preenchido (35)

Exclusão por forma TB

extrapulmonar e Pulmonar + extrapulmonar (595)

Exclusão por tipo de Entrada

recidiva, reingresso pós-

abandono, sem informação,

transferência, pós óbito (337)

\section{Exclusão por desfecho}

(situação de encerramento):

Mudança de diagnóstico, óbito

por outras causas, mudança de

esquema e transferência. (261)

Exclusão de duplicatas (3)

Fonte: Dados da pesquisa (2021).

Foram definidos como critérios de exclusão deste estudo notificações sem preenchimento do campo de situação de encerramento (excluídos 35), a forma de tuberculose extrapulmonar e a pulmonar combinada com extrapulmonar (excluídos 595), uma vez que para o campo da saúde pública a forma pulmonar, além de ser a mais frequente, é mais relevante por ser responsável pela manutenção da cadeia de transmissão da doença, especialmente quando bacilífera (Brasil, 2019a). Foram excluídos os casos com entrada especificadas no campo do formulário como de recidiva, reingresso pós abandono, "não sabe" que corresponde a ignorado, transferência, pós óbito (excluídos 337) por não constituírem casos novos diagnosticados.

Aplicando os critérios, foram excluídos 261 casos com a retirada das seguintes variáveis: mudança de diagnóstico por ter sido introduzida ao banco por erro diagnóstico ou de notificação, óbito por outras causas por não terem papel na análise epidemiológica do agravo e as variáveis mudança de esquema de tratamento e transferência por não oferecerem informações suficientes sobre o seguimento a não ser que fossem buscados caso a caso.

Com o uso do Excel foi realizada análise de duplicatas em que foram excluídas 3 notificações em duplicidade. Portanto, após aplicados os critérios de exclusão o estudo dos fatores associados a cura contou com 1237 notificações de casos novos de tuberculose pulmonar. 
O desfecho analisado, variável dependente, foi a evolução do caso, especificamente a cura, apresentada na variável situação de encerramento. Para tanto, foi considerado desfecho favorável a categoria "cura" e como desfecho desfavorável (não cura) as categorias: abandono, óbito por TB, tuberculose drogarresistente (TB-DR), falência de tratamento e abandono primário, que corresponde aos casos diagnosticados que não iniciaram tratamento.

Em seguida, as variáveis independentes foram escolhidas com base em um modelo hierárquico proposto por Maciel (2012). O modelo é composto por níveis interdependentes de vulnerabilidade com foco individual, contexto social e programática de assistência de saúde. No nível 1 considerou-se as variáveis sexo, escolaridade, raça/cor e idade; no nível 2, zona de residência, beneficiários de transferências governamentais e populações especiais (privada de liberdade, em situação de rua e profissionais de saúde); o nível 3, tabagismo, diabetes, doença mental e alcoolismo e o nível 4 tem como variáveis: realização de raio X, baciloscopia de diagnóstico, cultura de escarro, Teste Rápido Molecular e tratamento diretamente observado. Algumas variáveis de interesse foram incluídas no modelo hierárquico, adaptando-o conforme o próprio autor recomenda, pois são estruturas dinâmicas. Outras variáveis foram testadas conforme andamento do estudo, mas não foram incluídas.

Portanto, a variável desfecho foi associada com as seguintes variáveis independentes que assim foram categorizadas:

- sexo (masculino; feminino);

- idade (em faixas etárias: menor de 20; 20 a 39; 40 a 60; acima de 60);

- raça/cor da pele (branca; preta; parda; outras);

- escolaridade (analfabeto; ensino fundamental completo ou menos, ensino médio completo, ensino superior completo);

- zona de residência (urbana; rural;);

- beneficiários de transferências governamentais (sim; não);

- populações especiais (privada de liberdade, em situação de rua e profissionais da saúde);

- alcoolismo (sim; não);

- tabagismo (sim; não);

- diabetes (sim; não);

- doença mental (sim; não)

- tratamento diretamente observado (TDO) (sim; não);

- raio X realizado (sim; não);

- Teste rápido molecular realizado (sim; não);

- baciloscopia de escarro diagnóstica realizada (negativa ou positiva);

- Sorologia para HIV (negativa ou positiva) e

- cultura de escarro (negativa ou positiva);

Foi necessário o tratamento do banco de dados com a categorização de algumas variáveis e foi acrescentada a categoria "sem informação" para os campos sem informação das variáveis do SINAN.

A priori foi realizada análise descritiva das variáveis de interesse para o estudo (número absoluto e frequência). Para a análise bivariada foi empregado o teste do qui-quadrado de Pearson ou Exato de Fisher, quando valores esperados menores que 5 , para identificar as variáveis significativamente associadas com o desfecho $(\mathrm{p}<0,05)$. Foi realizada correção com post-hoc de teste $\mathrm{z}$ de Bonferroni para as variáveis politômicas. Os resultados foram descritos a partir de distribuição de frequências e análise de associação entre variáveis, assumindo-se significância de 5\% ( $<<0,05)$.

A análise bivariada foi realizada por regressão logística binária buscando verificar os fatores associados à cura da TB 
pulmonar e foram calculadas odds ratio (OR) e intervalos de confiança de 95\% (IC95\%) para todas as variáveis significativas $\mathrm{p}<0,20$. Embora o modelo final tenha comportado apenas duas variáveis significativas: diabetes e TDO (teste Hosmer and Lesmeshow com valor-p igual a 0,805), esta análise bivariada apresentou as razões de chances da fase anterior (triagem) para a avaliação das associações de cada preditor na resposta (cura/não cura). Os dados foram analisados pelo software SPSS Versão 20.0 e EPI INFO WEBSITE versão 7.2.4.0 https://www.cdc.gov/epiinfol.

Esta pesquisa foi aprovada pelo Comitê de Ética em Pesquisa da Universidade Federal dos Vales do Jequitinhonha e Mucuri sob parecer número 4.479.521 e CAAE 39902320.5.0000.5108, sendo respeitados todos os preceitos éticos.

\section{Resultados}

No período avaliado foram notificados no total 2468 casos de TB nos 53 municípios do Norte de Minas Gerais que fazem parte da pesquisa. Para o estudo dos fatores associados a cura da tuberculose pulmonar, após aplicados os critérios de inclusão e exclusão, restaram 1237 notificações de casos novos de tuberculose pulmonar, em que 1050 (84,9\%) foram encerrados por cura e $187(15,1 \%)$ com outros desfechos. A proporção de cura entre os casos novos de TB pulmonar com confirmação laboratorial no Brasil, em 2019, foi de 70,1\% (Brasil, 2021).

O estudo apresenta média de casos novos de TB pulmonar por ano de 123,7 $( \pm 16,53)$ com mínimo de 90 e máximo de 138 casos e percentual médio de cura de 84,97( $\pm 4,83)$ com mínimo de $74,6 \%$ e máximo de $90 \%$.

A maioria dos indivíduos acometidos são do sexo masculino 815 (65,9\%), pardos (63,3\%), com escolaridade igual ou inferior no ensino fundamental $520(67,1 \%)$ sem incluir $111(14,3 \%)$ indivíduos analfabetos e residentes na zona urbana 929 (79,5\%). Foi encontrada idade média de 55 anos ( \pm 19,7; mínimo :05 e máximo:104).

A Tabela 1 apresenta a associação entre a evolução dos casos de TB, cura e não cura, com as variáveis de interesse ao estudo.

Tabela 1 - Distribuição e fatores associados ao desfecho da tuberculose de acordo com o modelo hierárquico de eixos da vulnerabilidade, Norte de Minas Gerais, 2009-2018.

\begin{tabular}{|c|c|c|c|}
\hline Variáveis & $\begin{array}{l}\text { Cura } \\
\text { n }(\%)\end{array}$ & $\begin{array}{l}\text { Não cura } \\
\text { n }(\%)\end{array}$ & $(p \text {-valor })^{*}$ \\
\hline \multicolumn{4}{|l|}{ NÍVEL 1} \\
\hline $\operatorname{Sexo}(n=1237)$ & & & 0,003 \\
\hline Feminino & $376(89,1)$ & $46(10,9)$ & \\
\hline Masculino & $674(82,7)$ & $141(17,3)$ & \\
\hline $\operatorname{Raça}(n=1173)$ & & & 0,433 \\
\hline Branca & $204(86,8)$ & $31(13,2)$ & \\
\hline Preta & $155(86,6)$ & $24(13,4)$ & \\
\hline Parda & $624(83,6)$ & $122(16,4)$ & \\
\hline Outras & $10(76,9)$ & $3(23,1)$ & \\
\hline Escolaridade (n=775) & & & 0,018 ** \\
\hline Analfabeto ${ }^{\mathrm{A}}$ & $93(83,8)$ & $18(16,2)$ & \\
\hline $\begin{array}{l}\text { Ensino Fundamental completo ou } \\
\text { menos } \mathrm{AB}\end{array}$ & $440(84,6)$ & $80(15,4)$ & \\
\hline Ensino Médio completo ${ }^{\mathrm{AB}}$ & $109(94,0)$ & $7(6,0)$ & \\
\hline Ensino superior completo ${ }^{A}$ & $27(96,4)$ & $1(3,6)$ & \\
\hline Idade (n=1229) & & & $0,043 * *$ \\
\hline 0 a 19 АB & $24(100)$ & $0(0)$ & \\
\hline 20 a $39^{\mathrm{A}}$ & $247(87,6)$ & $35(12,4)$ & \\
\hline 40 a $59^{\mathrm{A}}$ & $353(82,3)$ & $76(17,7)$ & \\
\hline 60 ou mais ${ }^{A}$ & $419(84,8)$ & $75(15,2)$ & \\
\hline \multicolumn{4}{|l|}{ NIVEL 2} \\
\hline Zona de Residência (n=1169) & & & 0,727 \\
\hline Urbana & $793(85,4)$ & $136(14,6)$ & \\
\hline Rural & $207(86,2)$ & $33(13,8)$ & \\
\hline
\end{tabular}




\begin{tabular}{|c|c|c|c|}
\hline \multicolumn{3}{|l|}{$\begin{array}{l}\text { População privada de liberdade } \\
(n=546)\end{array}$} & \multirow[t]{3}{*}{0,089} \\
\hline Sim & $16(3,6)$ & $0(0)$ & \\
\hline Não & $433(96,4)$ & $97(100)$ & \\
\hline \multicolumn{4}{|l|}{$\begin{array}{l}\text { População em situação de rua } \\
(n=545)\end{array}$} \\
\hline Sim & $4(0,9)$ & $2(2,1)$ & 0,290 \\
\hline Não & $444(99,1)$ & $95(97,9)$ & \\
\hline \multicolumn{4}{|l|}{ Profissionais de saúde $(n=546)$} \\
\hline Sim & $3(0,7)$ & $0(0)$ & 1,000 \\
\hline Não & $445(99,3)$ & $98(100,0)$ & \\
\hline \multicolumn{4}{|l|}{$\begin{array}{l}\text { Beneficiários de transferências } \\
\text { governamentais }(n=452)\end{array}$} \\
\hline Sim & $42(11,3)$ & $2(2,5)$ & 0,016 \\
\hline Não & $330(88,7)$ & $78(97,5)$ & \\
\hline \multicolumn{4}{|l|}{ NIVEL 3} \\
\hline Tabagismo $(n=544)$ & & & 0,447 \\
\hline Sim & $69(15,4)$ & $18(18,6)$ & \\
\hline Não & $378(84,6)$ & $79(81,4)$ & \\
\hline \multicolumn{4}{|l|}{ Diabetes $(n=1142)$} \\
\hline Sim & $95(9,8)$ & $4(2,4)$ & $\mathbf{0 , 0 0 2}$ \\
\hline Não & $878(90,2)$ & $165(97,6)$ & \\
\hline \multicolumn{4}{|l|}{ Doença Mental $(n=1140)$} \\
\hline Sim & $32(3,3)$ & $7(4,1)$ & 0,576 \\
\hline Não & $939(96,7)$ & $162(95,9)$ & \\
\hline \multicolumn{4}{|l|}{ Alcoolismo $(n=1147)$} \\
\hline Sim & $167(72,9)$ & $62(27,1)$ & $<0,001$ \\
\hline Não & $808(88,0)$ & $110(12,0)$ & \\
\hline \multicolumn{4}{|l|}{ NIVEL 4} \\
\hline Realização de Raio X (n=1220) & & & 0,378 \\
\hline Sim & $929(89,9)$ & $171(91,9)$ & \\
\hline Não & $105(10,2)$ & $15(8,1)$ & \\
\hline Baciloscopia diagnóstica $(n=1061)$ & & & 0,752 \\
\hline Positiva & $741(82,1)$ & $128(81)$ & \\
\hline Negativa & $162(17,9)$ & $30(19)$ & \\
\hline \multicolumn{4}{|l|}{ Cultura $(n=266)$} \\
\hline Positiva & $169(74,4)$ & $31(79,5)$ & 0,501 \\
\hline Negativa & $58(25,6)$ & $8(20,5)$ & \\
\hline \multicolumn{4}{|l|}{ Realização de TRM (n=552) } \\
\hline Sim & $178(39,3)$ & $29(29,3)$ & 0,063 \\
\hline Não & $275(60,7)$ & $70(70,7)$ & \\
\hline \multicolumn{4}{|l|}{$\begin{array}{l}\text { Tratamento Diretamente Observado } \\
\text { realizado }(n=1049)\end{array}$} \\
\hline Sim & $684(75,0)$ & $91(66,4)$ & $\mathbf{0 , 0 3 3}$ \\
\hline Não & $228(25,0)$ & $46(33,6)$ & \\
\hline \multicolumn{4}{|l|}{ Sorologia HIV (n=710) } \\
\hline Positiva & $20(3,3)$ & $12(12,4)$ & $<0,001$ \\
\hline Negativa & $593(96,7)$ & $85(87,6)$ & \\
\hline
\end{tabular}

Nota: *Teste Qui-quadrado ou Exato de Fisher. ** Correção de Bonferroni ( $<<0,00625)$. ${ }^{\mathrm{AB}}$ Letras iguais significam similaridade entre as proporções do grupo. Fonte: Dados da pesquisa (2021).

Este estudo mostrou associação $(\mathrm{p}<0,05)$ entre os desfechos apresentados na variável encerramento e nove variáveis estudadas sendo, sexo, escolaridade, faixa etária, beneficiários de transferências governamentais, diabetes, alcoolismo, tratamento diretamente observado e sorologia para HIV conforme mostra a Tabela 1. As variáveis escolaridade e faixa etária foram submetidas a correção de Bonferroni por não serem variáveis dicotômicas e algumas categorias continuaram significativas após o post-hoc. 
A Tabela 2 apresenta os resultados da análise bruta do modelo de regressão logística binária, em que foram submetidas todas as variáveis significativas na fase anterior $(\mathrm{p}<0,20)$.

Tabela 2 - Análise bivariada por regressão logística binária dos fatores associados ao desfecho da tuberculose, Norte de Minas Gerais, 2009-2018.

\begin{tabular}{|c|c|c|c|}
\hline Variáveis & OR bruta & $I C(95 \%)$ & p-valor \\
\hline \multicolumn{4}{|l|}{$\operatorname{Sexo}(n=1237)$} \\
\hline Masculino & 1,00 & & \\
\hline Feminino & 1,71 & $1,198-2,441$ & 0,003 \\
\hline \multicolumn{4}{|l|}{ Escolaridade (n=775) } \\
\hline Analfabeto & 1,00 & & \\
\hline $\begin{array}{l}\text { Ensino Fundamental completo ou } \\
\text { menos }\end{array}$ & 1,06 & $0,609-1,860$ & 0,826 \\
\hline Ensino Médio completo & 3,01 & $1,206-7,531$ & 0,018 \\
\hline Ensino superior completo & 5,23 & $0,667-40,950$ & 0,115 \\
\hline \multicolumn{4}{|l|}{$\begin{array}{l}\text { Beneficiários de transferências } \\
\text { governamentais }(n=452)\end{array}$} \\
\hline Sim & 4,96 & $1,176-20,946$ & 0,029 \\
\hline Não & 1,00 & & \\
\hline \multicolumn{4}{|l|}{ Diabetes $(n=1142)$} \\
\hline Sim & 4,46 & $1,618-12,305$ & 0,004 \\
\hline Não & 1,00 & & \\
\hline \multicolumn{4}{|l|}{ Alcoolismo $(n=1147)$} \\
\hline Sim & 1,00 & & \\
\hline Não & 2,72 & $1,916-3,882$ & $<0,001$ \\
\hline \multirow{2}{*}{\multicolumn{4}{|c|}{ Observado realizado $(n=1049)$}} \\
\hline & & & \\
\hline Sim & 1,51 & $1,031-2,228$ & 0,034 \\
\hline Não & 1,00 & & \\
\hline \multicolumn{4}{|l|}{ Realização de TRM (n=552) } \\
\hline Sim & 1,56 & $0,974-2,505$ & 0,064 \\
\hline Não & 1,00 & & \\
\hline \multicolumn{4}{|l|}{ Sorologia para HIV $(n=710)$} \\
\hline $\operatorname{Sim}$ & 1,00 & & \\
\hline Não & 4,18 & $1,975-8,870$ & $<0,001$ \\
\hline
\end{tabular}

Fonte: Dados da pesquisa (2021).

Analisando o nível 1, neste estudo, os pacientes do sexo feminino apresentaram 1,71 (1,19-2,44) mais chances de cura do que os do sexo masculino. Observou-se maior chance de cura para os que apresentavam ensino médio completo em relação aos analfabetos (OR 3,01; IC95\% 1,21;7,53). A variável faixa etária quando disposta no modelo perdeu significância e apresentou outliers, portanto, não ajustou-se ao modelo regressão logística binária e foi retirada.

No nível 2, os pacientes que são beneficiários de transferências governamentais tiveram associado a maiores chances de cura (OR 4,96; IC95\% 1,17;20,94) se comparados aos que não recebem. Vale ressaltar que esta variável foi inserida no SINAN apenas em 2015 o que explica o número baixo de respostas. A variável população privada de liberdade não ajustou-se ao modelo de regressão e foi retirada. Nenhuma outra variável deste nível teve associação significativa. 
Entre as variáveis do nível 3 os não alcoolistas (OR 2,73; IC $95 \%$ 1,92;3,88) apresentaram mais chances de cura. No entanto, neste estudo, diabéticos apresentam 4,46 ( $\left.\mathrm{IC}_{95 \%} 1,61 ; 12,30\right)$ mais chances de cura da $\mathrm{TB}$ se comparados aos não diabéticos.

No nível 4 que aborda a assistência programática prestada ao usuário com TB apenas o tratamento diretamente observado (TDO) foi associado ao desfecho, sendo que os usuários que fizeram TDO apresentaram 1,51 (1,031-2,228) mais chances de cura se comparados aos que não fizeram. Os pacientes com sorologia para HIV negativa apresentam maiores chances de cura que os pacientes com sorologia positiva (OR 4,18; IC $95 \%$ 1,97;8,87).

\section{Discussão}

Na tuberculose pulmonar o encerramento por cura no Sistema de Informação se dá quando o paciente diagnosticado com TB pulmonar bacilífera, apresenta no decorrer do tratamento exames de baciloscopia de escarro negativas, sendo uma a qualquer momento do tratamento e outra nos dois últimos meses (Brasil, 2019b). A alta por cura também é conferida aos pacientes que completaram o tratamento, sem evidência de falência, considerando critérios clínicos, sendo estes conceitos os mesmos propostos pela OMS (2015).

A cura da TB está intrinsecamente relacionada a adesão ao tratamento, uma vez que, os fármacos aliados a cuidados integrais com a saúde podem combater os bacilos e quebrar a cadeia de transmissão da doença (Jesus, Souza, \& Silveira, 2012; Brasil, 2019b). O sucesso do tratamento depende da detecção precoce, do manejo correto e do protagonismo dos pacientes (Bertolozzi et al., 2014; Brasil, 2019b). Muitos são os fatores que interferem nessa dinâmica e esses podem orientar ações voltadas para a cura oportuna desses casos. No Brasil, existem políticas de acesso ao tratamento de TB pelo Sistema Único de Saúde (SUS), diferente de outras partes do mundo, mas há também um grande contingente vulnerável (Bertolozzi et al., 2014).

Para este estudo utilizamos a compreensão de vulnerabilidade que consideram as chances que um conjunto de fatores individuais, coletivos e sociais tem de influenciar no processo saúde-doença-cuidado. Tendo em vista os pressupostos da saúde coletiva e o modelo proposto por Maciel e Reis-Santos (2015) pode-se observar três dimensões: as dimensões individuais, sociais e programáticas, divididos aqui em quatro níveis.

Os achados deste estudo permitiram identificar os fatores associados a cura da TB pulmonar na região do norte de Minas Gerais em uma série histórica de 10 anos. Isto posto, ser do sexo feminino, ter ensino médio completo, ser beneficiário de transferências governamentais de renda, não ser HIV positivo, não ser alcoolista, fazer tratamento diretamente observado aumentam as chances de cura da TB. Contraditoriamente ao que se pode pensar o fato da pessoa ser diabético nesse estudo conferiu efeito positivo em relação à cura.

Em relação ao gênero o sexo feminino observou-se maior chance de cura da TB comparado ao sexo masculino. Este fato corrobora com os achados de estudo realizado no Rio de Janeiro em que o sexo masculino foi associado a menor chance de um desfecho favorável (OR 0,71; IC95\% 0,66;0,76), quando comparado ao sexo feminino (Santos et al., 2018).

Resultado semelhante também foi descrito em estudo que buscou comparar a proporção de cura por TB segundo a cobertura e o tempo de implantação de saúde da família e fatores socioeconômicos e demográficos no município do Rio de Janeiro a partir dos casos novos notificados no ano de 2012 que encontrou que pessoas do sexo feminino tinham 1,40 (IC95\% 1,21-1,62) vezes mais chance de cura de TB quando comparadas com pessoas do sexo masculino $(p<0,001)$ (Prado, Virgílio, \& Medronho, 2016). Contudo, estudo realizado na Paraíba com população privada de liberdade, 35 vezes mais suscetível ao adoecimento e majoritariamente masculina com $93,3 \%$ dos pacientes do sexo masculino, mostrou, no entanto, que não foi encontrada associação significativa entre a variável desfecho do tratamento e sexo, na análise bi e multivariada (Alves, Borralho, Araújo, Bernardino, \& Figueiredo, 2020). Essa diferença entre os sexos pode ter explicação na maior procura das mulheres aos serviços de saúde e maior acolhimento de suas necessidades relacionadas aos serviços de suporte a saúde da 
mulher e por terem melhor adesão aos tratamentos que os homens de maneira geral (Santos et al., 2018).

Sobre a escolaridade observou-se, neste estudo, três vezes mais chances de cura quando comparados os que apresentavam ensino médio completo em relação aos analfabetos (OR 3,01; IC95\% 1,21;7,53) corroborando com os achados de estudo realizado no Somália que associou pacientes analfabetos e apenas com o ensino fundamental a uma menor chance de resultado de tratamento bem sucedido em comparação com pacientes com ensino médio OR 4,1, IC 95\% 1 a 15,9 e OR 5,9, IC 95\% 1,6 a 21,8, respectivamente (Ali, Karanja, \& Karama, 2017).

Estudos brasileiros mostram que pessoas com nível superior têm 2,10 (IC95\% 1,43-3,08) vezes mais chance de cura quando comparadas com pessoas analfabetas ( $p<0,001)$ (Prado et al., 2016), e ainda, associam maior chance de desfecho favorável entre aqueles com 9-12 anos de estudo (OR 2,04; IC95\% 1,68;2,47) e 13 anos ou mais de estudo (OR 3,48; IC95\% 2,74;4,41), relativamente aos analfabetos (Santos et al., 2018). Estudo realizado em Salvador mostrou maior proporção de cura entre participantes com escolaridade superior a nove anos, vivendo em união estável e com baixa densidade de pessoas por domicílio (Andrade, Nery, Araújo, Barreto, \& Pereira, 2019). Explicado pelo fato de que o autocuidado/adesão é comprometido na baixa escolaridade e pelo fato de estar ligada a piores condições de vida e, consequentemente, de maior vulnerabilidade (Jesus et al., 2012).

Os pacientes que são beneficiários de transferências governamentais estavam associados a maiores chances de cura (OR 4,96; IC95\% 1,17;20,94) se comparado aos que não recebem. Percebe-se semelhança aos resultados de estudo randomizado não cego realizado em 32 favelas no Peru que objetivou avaliar o impacto do suporte socioeconômico no início da terapia preventiva da TB em contatos domiciliares de pacientes com TB e no sucesso do tratamento nos pacientes. Este estudo demonstrou que a intervenção (aporte econômico) foi associada a um aumento na taxa de sucesso do tratamento em ambos os subgrupos mais pobres e menos pobres. Além disso, a intervenção aumentou significativamente o início da terapia preventiva em contatos com menos de cinco anos (OR: 2,2; IC 95\%: 1,1-4,2) (Wingfield et al., 2017).

Recentemente, os resultados de uma meta análise mostrou que estratégias de proteção social em países de baixa ou média renda estão associadas a cura da TB ( $\mathrm{RR}=1,11$; IC 95\%: 1,01-1,22) e com redução do risco de abandono do tratamento da TB ( $R R=0,63$; IC 95\%: 0,45-0,89) reforçando as premissas de que os fatores sociodemográficos e socioeconômicos impactam no tratamento da TB, principalmente melhorando sua adesão (Andrade et al., 2018). No Brasil, estudo realizado em Salvador, o primeiro a pesquisar sobre os benefícios sociais e o impacto no tratamento da $\mathrm{TB}$ mostrou que existe maior proporção de cura entre os indivíduos que recebem benefícios governamentais e não governamentais durante o tratamento de TB (Andrade et al., 2019).

Certamente o Programa Nacional de Controle da Tuberculose (PNCT) deve dar prioridade às políticas públicas que atuem neste sentido, melhorando as coberturas de saúde e transferências de renda vinculadas as ações estratégicas, para que ocorra o alcance das metas de eliminação da tuberculose como problema de saúde pública no Brasil. Ressalta-se que o escopo da proteção social é por vezes muito amplo, incluindo intervenções financeiras e não financeiras, tais como, apoio econômico, psicológico, nutricional, educação em saúde e mobilização social (Andrade et al., 2018).

Neste estudo, apresentaram maiores chances de cura os pacientes que são HIV negativos no exame sorologia (OR 4,18; IC $95 \%$ 1,97;8,87) em comparação aos HIV positivo, esse achado corrobora com o estudo realizado no Rio de Janeiro, 2011 a 2014, que demonstrou associação em ser HIV positivo e reduzir as chances de sucesso no resultado do tratamento (OR 4,4, IC 95\% 1,1 a 17,7) em comparação com os pacientes HIV negativos (Santos et al., 2018) e estudo realizado com a população privada de liberdade na Paraíba que verificou associação estatisticamente significativa entre os desfechos (cura ou abandono) e a AIDS ( $p=0,044)$ (Alves, Borralho, Araújo, Bernardino, \& Figueiredo, 2020). A coinfecção HIV/TB é um grande problema de saúde pública, responsável por grande contingente de óbitos (Brasil, 2019b; Brasil, 2021). Globalmente, dados recentes mostram taxas de sucesso de $85 \%$ para TB e $76 \%$ para pacientes vivendo com HIV (PVHIV), sendo que nas 
Américas esse valor ficou em torno de 56\% em 2018 (OMS, 2020).

O tratamento da TB em PVHIV obedece às mesmas recomendações em relação à população geral, tanto na utilização dos fármacos quanto na duração total do tratamento. A TB é curável em quase a totalidade dos casos também nessa população, porém falhas ou intercorrências ao longo do tratamento podem implicar maior risco de abandonos e óbitos (Brasil, 2019b).

Por outro lado, os pacientes não alcoolistas apresentaram 2,7 mais chances de cura que os alcoolistas neste estudo. $O$ que reforça que o consumo de álcool pode comprometer a adesão, o autocuidado e levar a outras fragilidades sociais e de rede de apoio familiar (Brasil, 2019b). Nos EUA, há relatos de aumento da incidência de 9 a 55 vezes maior de TB entre usuários de álcool e aumento do curso desfavorável da doença (Brasil, 2019b). No ano de 2018, o percentual de cura da doença entre os casos novos confirmados por critério laboratorial que referiram uso de álcool foi de $65 \%$ e o abandono de $17 \%$, na população geral os resultados são de 75\% e 11\% (Brasil, 2019b). Ademais outros estudos demonstram que os alcoolistas apresentaram probabilidade quase quatro vezes maior de abandonar o tratamento (Silva, dos Santos Lafaiete, \& Donato, 2011). Estudo de revisão sistemática da literatura sobre a tuberculose e indicadores socioeconômicos apontou em análise multivariada que os desfechos negativos tinham associação direta com a baixa escolaridade, coinfecção com HIV e alcoolismo que corroboram com os achados deste estudo (San Pedro \& Oliveira, 2013).

Sumariamente, o tratamento diretamente observado (TDO) é uma ação altamente recomendada no controle da TB em todo o mundo, sendo uma das ações pactuadas pela OMS para o alcance da meta global pelo fim da tuberculose com redução da incidência para 10 por 100 mil habitantes até 2035 (Bertolozzi et al., 2014; Brasil, 2021). O TDO consiste em que semanalmente, pelo menos durante três dias as doses diárias da medicação para tratamento da TB sejam tomadas sob observação de um profissional da atenção primária à saúde, na Unidade Básica de Saúde ou na casa ou local de trabalho do paciente (Brasil,2019b).

Esta ação programática foi associada ao desfecho cura neste estudo, sendo que os usuários que fizeram TDO apresentam 1,51 (1,031-2,228) mais chances de cura se comparados aos que não o fizeram. Este resultado vem corroborar com revisão sistemática da literatura dos pesquisadores San Pedro e Oliveira (2013) que apontam que a mortalidade por TB foi associada dentre outras variáveis a não ter recebido tratamento diretamente observado (TDO). Tal qual em outro estudo brasileiro conduzido por Maciel e Reis-Santos (2015) que atribui maiores chances de cura aos indivíduos com tratamento diretamente observado se comparados a todos os outros desfechos.

Apesar da diabetes ser considerada pela OMS (2017) um dos cinco fatores de risco para a TB, os pacientes diabéticos, deste estudo, apresentam 4,46 ( IC $_{95 \%}$ 1,61;12,30) mais chances de cura da TB se comparados aos não diabéticos. Este achado vem em contraposição ao observado na literatura que denota que a diabetes mellitus retarda a resposta microbiológica podendo resultar em menores taxas de cura, ocorrência de recidivas e possibilidade maior para desenvolver as formas resistentes (Brasil, 2019b).

Nesse sentido, são de fundamental importância o manejo correto e o acompanhamento constante deste paciente o que pode resultar por outro lado em melhores taxas de cura. Interroga-se o fato do paciente diabético já ser usuário diário de medicação levaria a uma maior adesão ao tratamento da TB. Esta hipótese corrobora com resultados de uma Pesquisa Nacional de Saúde realizada em 2013, que obteve que 73,2\% dos diabéticos referiram terem sido atendidos nos últimos 12 meses, 47,1\% em Unidade Básica de Saúde do SUS o que demostra uma boa procura aos serviços de saúde por esta população (Brasil, 2019b). Inclusive estudos apontam que a presença da atenção primária repercute positivamente na adesão ao tratamento e consequentemente para desfechos favoráveis (Andrade et al., 2018; Andrade et al., 2019). Estudo realizado no Brasil nos anos de 2007 a 2011 analisou 318.465 casos de TB e detectou que a coinfecção de diabetes e TB está associada a melhores taxas de adesão ao tratamento (OR 0,68; IC 95\% = 0,64-0,73) (Maciel \& Reis-Santos, 2015).

Apesar de não se apresentarem significativas nesta população estudada, algumas variáveis oferecem em outros 
estudos resultados diferentes. Por exemplo, existe diferenças significativas quanto a variável raça/cor em estudo realizado no Rio de Janeiro em que indivíduos da raça/cor da pele preta (OR 0,62; IC95\% 0,57;0,67) e parda (OR 0,73; IC95\% 0,68;0,78) foram associadas a menor chance de cura, comparados àqueles de raça/cor branca; a mesma associação se observou na faixa etária de 20-39 anos (OR 0,65; IC95\% 0,58;0,73) (Santos et al., 2018). Estudo transversal no Estado do Rio Grande do Sul mostrou proporção de cura maior entre os indivíduos brancos (73\%) em relação aos indivíduos negros (16\%) (Deus et al., 2020).

Em outro estudo realizado em Salvador, que tem sua maioria afrodescendente, a proporção de cura também é maior em brancos, apesar da prevalência ser maior na população negra e parda. No entanto, tal qual neste estudo, essas diferenças não foram estatisticamente significativas (Andrade et al., 2019).

Outra variável importante é a faixa etária, que em alguns estudos encontram associações com o desfecho de cura apresentando menor chance de cura adultos na faixa etária de 20-39 anos (OR 0,65; IC95\% 0,58;0,73) (Santos et al., 2018). Neste estudo, apesar de não ser adequados, para cálculos estatísticos, nota-se que 100\% dos indivíduos acometidos pela TB na faixa etária de 0 a 19 anos tiveram desfecho favorável. O Boletim Global da Tuberculose de 2020 mostra uma taxa de cura para crianças e adolescentes jovens de $84 \%$ bem semelhante à dos adultos (OMS, 2020). Em estudo realizado no Rio Grande do Sul o maior percentual de cura ocorreu na faixa etária 0 a 14 anos $(89 \%)$, sendo fator protetor para a cura da TB o que pode ser atribuído ao fato dos cuidados familiares serem maiores nessa faixa etária bem como a menor probabilidade de resistência ao fármaco (Deus et al., 2020).

Quanto às populaçães mais vulneráveis, tais como população privada de liberdade e população vivendo em situação de rua, ressalta-se que são notáveis alvos de estratégias de enfrentamento da TB por apresentarem risco maior de adoecimento e desfechos desfavoráveis, porém neste estudo não apresentaram diferenças significativas. No estudo do RJ, no entanto, situação de institucionalizado em asilo (OR 0,35; IC95\% 0,15;0,83) e em outros estabelecimentos diferentes de presídio, asilo, orfanato e hospital psiquiátrico (OR 0,40; IC95\% 0,32;0,49) apresentaram menores chances de um desfecho favorável, quando comparadas a não estar institucionalizado (Santos et al., 2018). Em pesquisa realizada com população privada de liberdade na Paraíba, observou-se associação estatisticamente significativa entre os desfechos (cura ou abandono) e a AIDS ( $p=0,044$ ), a sorologia para HIV $(p=0,048)$ e a não realização de baciloscopia de acompanhamento $(p=0,001)$, fazendo perceber que podem ocorrer limitações no diagnóstico de comorbidades importantes e no acompanhamento nesses espaços (Alves et. $a l, 2020)$. Nota-se aqui a possibilidade de ocorrência de dificuldades diagnósticas próprias das condições de vida.

O estudo apresentou limitações relacionadas as incompletitudes das variáveis, informações ignoradas ou ausentes. Essas são características já esperadas aos estudos que utilizam dados secundários advindos dos sistemas de informação (Pinheiro, Andrade, \& Oliveira, 2012; Barbosa, Barrado, Zara, \& Siqueira Júnior, 2015). No entanto, a quantidade de notificações deste estudo perfaz poder estatístico suficiente para detectar as diferenças entre os grupos. Outra limitação encontrada foi adequação do modelo de Regressão Logística Binária para apenas duas variáveis estudadas.

\section{Considerações Finais}

O presente estudo foi realizado em uma região marcada por grandes desigualdades sociais e indicadores de desenvolvimento sociais ruins, que tem uma das maiores cargas de tuberculose do Estado de Minas Gerais (Minas Gerais, 2019) e abarcando uma população superior a um milhão de habitantes por um período de 10 anos e revelou que a cura da tuberculose está associada a fatores socioeconômicos e de assistência corroborando com o modelo teórico de eixos de vulnerabilidades. Evidenciou, que nesta população estudada, ser do sexo feminino, ter ensino médio completo, ser beneficiário de transferências governamentais de renda, não ser HIV positivo, não ser alcoolista, ser diabético, fazer tratamento diretamente observado aumentam as chances de cura da TB pulmonar. 
Doravante, conhecer os aspectos associados à cura da TB e os determinantes da não cura, que são os desfechos desfavoráveis do enfrentamento da TB, é reconhecer a emblemática determinação social dessa doença no território. É preciso políticas públicas com foco na redução da pobreza, no aumento do acesso a saúde, na proteção social, políticas que envolvam toda a sociedade.

À luz do que foi abordado ao longo deste estudo, recomenda-se, veementemente, à vigilância epidemiológica o correto preenchimento das fichas de notificação e acompanhamento com o objetivo de melhorar a qualidade dos bancos de dados sendo evidente a necessidade de processos formativos de educação permanente em saúde para os profissionais.

Espera-se, portanto, reforçar os esteios e pilares que devem ser fundamentos para ações multisetoriais nos mais diversos e complexos eixos da proteção social desta população vulnerável adoecida, a fim de envidar esforços na busca de soluções arrojadas para tão fortes aspectos de desigualdades sociais, históricos e contínuos perpetuadores da tuberculose no Brasil.

\section{Referências}

Ali, M. K., Karanja, S., \& Karama, M. (2017). Factors associated with tuberculosis treatment outcomes among tuberculosis patients attending tuberculosis treatment centres in 2016-2017 in Mogadishu, Somalia. Pan African Medical Journal, 28(1).

Alves, K. K. A. F., Borralho, L. M., Araújo, A. J. de., Bernardino, Í. de M., \& Figueiredo, T. M. R. M. de. (2020). Fatores associados à cura e ao abandono do tratamento da tuberculose na população privada de liberdade. Revista Brasileira de Epidemiologia, 23, e200079.

Andrade, K. V. F. D., Nery, J. S., Araújo, G. S. D., Barreto, M. L., \& Pereira, S. M. (2019). Associação entre desfecho do tratamento, características sociodemográficas e benefícios sociais recebidos por indivíduos com tuberculose em Salvador, Bahia, 2014-2016. Epidemiologia e Serviços de Saúde, 28.

Andrade, K. V. F. D., Nery, J. S., Souza, R. A. D., \& Pereira, S. M. (2018). Effects of social protection on tuberculosis treatment outcomes in low or middleincome and in high-burden countries: systematic review and meta-analysis. Cadernos de Saúde Publica, $34, \mathrm{e} 00153116$.

Barbosa, J. R., Barrado, J. C. D. S., Zara, A. L. D. S. A., \& Siqueira Júnior, J. B. (2015). Avaliação da qualidade dos dados, valor preditivo positivo, oportunidade e representatividade do sistema de vigilância epidemiológica da dengue no Brasil, 2005 a 2009. Epidemiologia e Serviços de Saúde, 24, 49-58.

Bertolozzi, M. R., Takahashi, R. F., Hino, P., Litvoc, M., \& de Siqueira França, F. O. (2014). O controle da tuberculose: um desafio para a saúde pública. Revista de Medicina, 93(2), 83-89.

Campos, P. F.S., \& de Silva, D. N. da (2020). História de la salud: tuberculosis en las cartas a Esther (São Paulo, 1905-1919). Cultura de los Cuidados (Edición digital), 24, 57.

Deus, A. P. L., Goerch, H. G. C., Noal, H. C., Megier, E. R., \& Anversa, E. T. R. (2020). Tratamento e abandono de casos notificados de Tuberculose do Estado do Rio Grande do Sul. Research, Society and Development, 9(9), e669997659-e669997659.

Galvão, T. F., \& Pereira, M. G. (2014). Revisões sistemáticas da literatura: passos para sua elaboração. Epidemiologia e Serviços de Saúde, $23,183-184$.

Instituto Brasileiro de Geografia e Estatística - IBGE (2020). Rio de Janeiro. https://www.ibge.gov.br.

Jesus, B. F. G. D., Souza, P. G. O., \& Silveira, M. F. (2012). Espirito Santo LR. Perfil epidemiológico de tuberculose na cidade de Montes Claros de 2005 a 2009. Rev Bras Farm, 93(1), 80-84.

Maciel, E. L. (2012). Determinantes sociais da tuberculose: elementos para a ação. Promoção da saúde na diversidade humana e nos itinerários terapêuticos. Campinas: Saberes.

Maciel, E. L., \& Reis-Santos, B. (2015). Determinants of tuberculosis in Brazil: from conceptual framework to practical application. Revista Panamericana de Salud Pública, 38, 28-34.

Malta, M., Cardoso, L. O., Bastos, F. I., Magnanini, M. M. F., \& Silva, C. M. F. P. D. (2010). Iniciativa STROBE: subsídios para a comunicação de estudos observacionais. Revista de Saúde Pública, 44(3), 559-565.

Ministério da Saúde, Secretaria de Vigilância em Saúde (2021). Vol. Especial. Brasília. Boletim Epidemiológico da Tuberculose. https://www.gov.br/saude/ptbr/media/pdf/2021/marco/24/boletim-tuberculose-2021_24.03

Ministério da Saúde. Secretaria de Vigilância em Saúde. (2019a). Brasil livre da Tuberculose: evolução dos cenários epidemiológicos e operacionais da doença. Boletim Epidemiológico (09).

Ministério da Saúde. Secretaria de Vigilância em Saúde (2019b). Recomendações para o Controle da Tuberculose no Brasil [Manual]. Brasília: Departamento de Vigilância das Doenças Transmissíveis. 364 p.

Minas Gerais. Secretaria de Estado de Saúde (2019). Plano estadual pelo fim da tuberculose como problema de saúde pública em Minas Gerais 2019-2022. https://www.saude.mg.gov.br/images/noticias_e_eventos/000_2019/jane_fev_mar/PLANO_ESTADUAL_PELO_FIM_DA_TUBERCULOSE_COMO_PROB LEMA_DE_SA\%c3\%9aDE_P\%c3\%9aBLICA_EM_MG.pdf 
Research, Society and Development, v. 10, n. 14, e544101422490, 2021

(CC BY 4.0) | ISSN 2525-3409 | DOI: http://dx.doi.org/10.33448/rsd-v10i14.22490

Organização Mundial da Saúde (2015). Relatório global da tuberculose 2015, edição 20. https://apps.who.int/iris/handle/10665/191102.

Organização Mundial da Saúde (2017). Relatório $\quad$ global $\quad$ da $\quad$ tuberculose, $2017 . \quad 147 \quad$ p https://www.who.int/tb/publications/global_report/gtbr2017_main_text.pdf.

Organização Mundial da Saúde (2020). Relatório global da tuberculose, 2020. 232 p. https://www.who.int/publications/i/item/9789240013131.

Pinheiro, R. S., Andrade, V. D. L., \& Oliveira, G. P. D. (2012). Subnotificação da tuberculose no Sistema de Informação de Agravos de Notificação (SINAN): abandono primário de bacilíferos e captação de casos em outras fontes de informação usando linkage probabilístico. Cadernos de Saúde Pública, 28, 15591568 .

Pinto, P. F. P. S., Silveira, C., Rujula, M. J. P., Chiaravalloti, F., \& Ribeiro, M. C. S. D. A. (2017). Perfil epidemiológico da tuberculose no município de São Paulo de 2006 a 2013. Revista Brasileira de Epidemiologia, 20, 549-557.

Prado, J. C., Virgilio, T. C., \& Medronho, R. D. A. (2016). Comparação da proporção de cura por tuberculose segundo cobertura e tempo de implantação de Saúde da Família e fatores socioeconômicos e demográficos no município do Rio de Janeiro, Brasil, em 2012. Ciência \& Saúde Coletiva, 21, 1491-1498.

San Pedro, A., \& Oliveira, R. M. D. (2013). Tuberculose e indicadores socioeconômicos: revisão sistemática da literatura. Revista Panamericana de Salud Pública, 33, 294-301.

Santos, J. N. D., Sales, C. M. M., Prado, T. N. D., \& Maciel, E. L. (2018). Fatores associados à cura no tratamento da tuberculose no estado do Rio de Janeiro, 2011-2014. Epidemiologia e Serviços de Saúde, 27.

Silva, C. B., dos Santos Lafaiete, R., \& Donato, M. (2011). O consumo de álcool durante o tratamento da tuberculose: percepção dos pacientes. SMAD, Revista Electrónica en Salud Mental, Alcohol y Drogas, 7(1), 10-17.

Silva, P. D. F., Moura, G. S., \& Caldas, A. D. J. M. (2014). Factors associated with pulmonary TB treatment dropout in Maranhão State, Brazil, from 2001 to 2010. Cadernos de saude publica, 30(8), 1745-1754.

Sousa, G. F., Mendes, A. L. R., Carvalho, G. D., Melo, S. M., \& de Araujo Carvalho, R. M. (2021). Perfil epidemiológico da tuberculose no Estado do Piauí no período de 2015 a 2020. Research, Society and Development, 10(9), e34310918150-e34310918150.

Valente, B. C., Angelo, J. R., Kawa, H., \& Baltar, V. T. (2019). A tuberculose e seus fatores associados em um município da região metropolitana do Rio de Janeiro. Revista Brasileira de Epidemiologia, 22, e190027.

Vilela, A. F. R., Melo, G., Neves, F. C. S., dos Reis, G. A. S., Lima, G. M., de Castro Monteiro, G., \& Sampaio, R. A. (2021). Prevalência e desfecho da tuberculose no Estado de Goiás. Research, Society and Development, 10(11), e556101119869-e556101119869.

Wingfield, T., Tovar, M. A., Huff, D., Boccia, D., Montoya, R., Ramos, E., Datta, S., Saunders, M. J., Lewis, J. J., Gilman, R. H., \& Evans, C. A. (2017). A randomized controlled study of socioeconomic support to enhance tuberculosis prevention and treatment, Peru. Bulletin of the World Health Organization, 95(4), 270. 